\title{
Polarization resolution of LISA
}

\author{
O Jennrich $\dagger$, M Peterseim $\dagger$, K Danzmann $\dagger \ddagger$ and B F Schutz $\S \uparrow$ \\ $\dagger$ Institut für Atom- und Molekülphysik, Universität Hannover, Appelstraße 2, 30167 Hannover, \\ Germany \\ $\ddagger$ Max-Planck-Institut für Quantenoptik, Hans-Kopfermann-Straße 1, 85748 Garching, Germany \\ $\S$ University of Wales, Cardiff College, Cardiff, Wales \\ I Albert-Einstein-Institut, Max-Planck-Institut für Gravitationsphysik, Potsdam, Germany
}

Received 13 August 1996, in final form 13 January 1997

\begin{abstract}
We discuss LISA's ability to resolve different polarizational states of a gravitational wave with fixed frequency and amplitude. Assuming a binary as the source of the gravitational wave, its orientation is connected with the polarization of the gravitational wave emitted. Using methods of signal processing, we calculate the $1-\sigma$ uncertainty range for measuring the orientation of the source.
\end{abstract}

PACS numbers: $9780 \mathrm{G}, 9585 \mathrm{~S}, 0480 \mathrm{~N}, 9555 \mathrm{Y}$

\section{Introduction}

Neutron star binaries are believed to be a copious source of gravitational waves in the frequency interval from $0.1-100 \mathrm{mHz}$ [3], well within the range of LISA's best sensitivity. Emitting in this frequency range, coalescence of such a binary will take place in the far future. Its dynamics is mainly governed by Newtonian mechanics [7] and its frequency will remain constant within the typical duration of a measurement performed by LISA, i.e. a few years.

Apart from determining the position $[1,2]$ and the frequency of the source, measuring the polarization state of a gravitational wave is of fundamental interest, as it is related to the orientation of the emitting binary.

\section{Signal from a binary}

Consider a binary consisting of two pointlike masses $m_{1}$ and $m_{2}$. As the power emitted in gravitational waves is small, it is reasonable to treat the signal as monochromatic in the source reference frame.

For such a system, having total mass $M=m_{1}+m_{2}$ and reduced mass $\mu=m_{1} m_{2} / M$, the tensor $\boldsymbol{h}^{t t}$ representing the gravitational wave, is of the form [7]

$$
\boldsymbol{h}^{t t}=\frac{4 \mu}{R}(M \omega)^{2 / 3} \Re\left\{\left(\begin{array}{cc}
h_{+} & h_{\times} \\
h_{\times} & -h_{+}
\end{array}\right) \exp \left[\mathrm{i} \omega_{\mathrm{g}}(z-t)\right]\right\} \quad \omega_{\mathrm{g}}=2 \omega
$$

with the amplitudes $h_{+}$and $h_{\times}$of the plus- and cross-polarization, respectively. Note that the frequency of the gravitational wave $\omega_{\mathrm{g}}$ is twice the angular velocity $\omega$, i.e. frequency of the binary. 
To cover different orientations of the binary we have introduced the usual spherical coordinates $(\cos \beta, \alpha)$ for the angular momentum of the binary, $\beta$ denoting the angle enclosed by the angular momentum and the $z$-axis, $\alpha$ denoting the angle enclosed by the projection of the angular momentum onto the $x-y$-plane and the $x$-axis.

This leads to a gravitational wave propagating along the $z$-axis with amplitudes

$$
\begin{aligned}
& h_{+}=\frac{1}{2}\left(\cos ^{2} \beta+1\right) \cos 2 \alpha+\mathrm{i} \cos \beta \sin 2 \alpha \\
& h_{\times}=\frac{1}{2}\left(\cos ^{2} \beta+1\right) \sin 2 \alpha-\mathrm{i} \cos \beta \cos 2 \alpha .
\end{aligned}
$$

Equation (2) shows a fourfold symmetry in $\alpha$, as $\alpha$ can be replaced by one of $\alpha+\pi / 2, \alpha+$ $\pi, \alpha+(3 \pi) / 2$ without changing (2) up to an overall sign.

Choosing the angular momentum aligned with the $z$-axis, i.e. $\beta=\alpha=0$ leads to a circularly polarized gravitational wave, whereas aligning the angular momentum with the $x$-axis or the bisector of the $x$ - and $y$-axes $(\beta=\pi / 2, \alpha=0$ or $\alpha=\pi / 4)$ gives a linearly plus polarized and a linearly cross polarized gravitational wave, respectively.

Hence, $\beta$ controls the type of polarization, circular or linear, whereas $\alpha$ chooses between plus or cross linear polarized and left or right circular polarized, respectively.

\section{Detector's response}

As the signal is defined with respect to the centre of mass of the source, it is necessary to transform the signal into the frame of the detector system in order to construct the detector's response.

We have chosen to transform $\boldsymbol{h}^{t t}$ by means of a matrix $\boldsymbol{D}(t ; \theta, \varphi)$ which includes the dependence of the location of the source, given by $\theta$ and $\varphi$ [1]. Its time dependence is due to the non-isotropy of LISA's sensitivity pattern, which gives rise to an amplitude modulation of the signal.

In addition, the gravitational wave in the detector system suffers a phase-shift varying with time due to the Doppler effect, described by a time-dependent phase $\Phi(t)$. Thus, $\boldsymbol{H}$ representing the transformed $\boldsymbol{h}^{t t}$ is

$$
\boldsymbol{H}=\Re\left\{\boldsymbol{D}^{T}(t ; \theta, \varphi) \boldsymbol{h}^{t t} \boldsymbol{D}(t ; \theta, \varphi) \exp \left[\mathrm{i}\left(\vec{k}_{\mathrm{g}} \vec{x}-\omega_{\mathrm{g}} t+\Phi(t)\right)\right]\right\}
$$

the wavevector $\vec{k}_{\mathrm{g}}$ being defined in the detector system.

Again following the arguments of [1] the signal $m$ obtained by LISA is given by

$$
m=\pi \frac{L}{\lambda} \sqrt{3}\left(H_{12}-\sqrt{3} H_{11}\right)
$$

with $L$ and $\lambda$ denoting LISA's arm length and the wavelength of the light used and $H_{12}$ and $H_{11}$ being the components of $\boldsymbol{H}$.

\section{Gaussian approximation}

To obtain results concerning LISA's ability to distinguish different orientations of a binary, we made use of methods of signal processing and extraction presented in [4].

The probability $P(\vec{\mu} \mid s) \mathrm{d} \vec{\mu}$ to find a parametrization within the interval $[\vec{\mu}, \vec{\mu}+\mathrm{d} \vec{\mu}]$ for a noisy signal $s(t ; \vec{\mu})=m(t ; \vec{\mu})+n(t)$ is Gaussian for sufficiently high signal-to-noise ratios $\rho[6]$.

Given a constant a priori probability for all degrees of freedom, i.e. components of the vector $\vec{\mu}$, the Gaussian probability is centred around the 'true' parametrization $\vec{\mu}_{0}$ for the 
signal $s$, i.e. the parametrization that matches the pure signal $m\left(t, \vec{\mu}_{0}\right)$ and the covariance matrix $\sigma_{i j}^{2}$ is given by

$$
\sigma_{i j}^{2}=C_{i j}
$$

The matrix $C^{-1}$ is called the Fisher matrix and is defined by means of the inner product of the derivatives of the signal.

$$
C_{i j}^{-1}=2\left\langle\partial_{i} s(t ; \vec{\mu}), \partial_{j} s(t ; \vec{\mu})\right\rangle .
$$

The shorthand notation $\partial_{i}$ means the derivative with respect to the $i$ th component of the vector $\vec{\mu}$.

The inner product of two functions is for these purposes defined as a convolution weighted with the expected spectral density $\mathcal{S}_{\mathrm{n}}(f)$ of the noise (matched filtering) [5] performed in Fourier space.

$$
\langle a, b\rangle=\int_{0}^{\infty} \mathrm{d} f \frac{\tilde{a}(f) \tilde{b}^{*}(f)+\tilde{a}^{*}(f) \tilde{b}(f)}{\mathcal{S}_{\mathrm{n}}(f)} .
$$

The relation of the quadratic spectral density of the noise $\mathcal{S}_{\mathrm{n}}(f)$ and $\rho$ is given by $[6,7]$

$$
\rho^{2} \approx 2\langle m(\vec{\mu}), m(\vec{\mu})\rangle .
$$

Having a signal which is concentrated within a small frequency interval, $\mathcal{S}_{\mathrm{n}}(f)$ can be considered as constant over this frequency interval. Thus equation (6) can be written as

$$
C_{i j}^{-1}=\rho^{2} \frac{\int_{0}^{\infty} \mathrm{d} f \partial_{i} \tilde{m} \partial_{j} \tilde{m}^{*}+\partial_{i} \tilde{m}^{*} \partial_{j} \tilde{m}}{\int_{0}^{\infty} \mathrm{d} f|\tilde{m}|^{2}} .
$$

The 'error volume' $\Sigma$ is given by the determinant of the covariance matrix,

$$
\Sigma=\sqrt{\operatorname{det} C}=\frac{1}{\sqrt{\operatorname{det} C^{-1}}}
$$

thus, from (9) it is easily obtained that

$$
\Sigma \sim \rho^{-n}
$$

where $n$ is the dimension of $\vec{\mu}$ and hence the dimension of the parameter space.

\section{Application to polarization}

In the case of the angular momentum of a binary the vector $\vec{\mu}$ consists of the two entries $(\cos \beta, \alpha)$ and the 'error volume' $\Sigma$ is usually denoted as the solid angle $\mathrm{d} \Omega$,

$$
\Sigma=\mathrm{d} \Omega=\mathrm{d} \alpha \mathrm{d} \beta \sin \beta
$$

assuming $\alpha$ and $\cos \beta$ to be uncorrelated. The meaning of $\mathrm{d} \Omega$ is the following: given a unit vector $\vec{n}$ pointing along the angular momentum of the binary, the top of $\vec{n}$ will move within the area $\mathrm{d} \Omega$ while tilting the plane of rotation of the binary slightly.

Hence, $d \Omega$ is a measure for the accuracy with which the orientation of the binary can be detected. 


\section{Results}

All the results presented in the following are obtained by using a fixed signal-to-noise ratio $\rho=100$ as it seems to be reasonable for signals expected in the frequency range around $3 \mathrm{mHz}$. Moreover, we restricted ourselves to a monochromatic source with a frequency $f_{\mathrm{g}}$ of $3 \mathrm{mHz}$.

Figure 1 shows the orientation error as a function of the polarization state for various source positions. The maximum and minimum curves belong to different values of $\alpha$, hence different initial phases. Whereas the solid angle is close to $100 \mu \mathrm{sr}$ for circularly polarized gravitational waves, it varies by three orders of magnitude for linearly polarized gravitational waves for sources close to the poles.

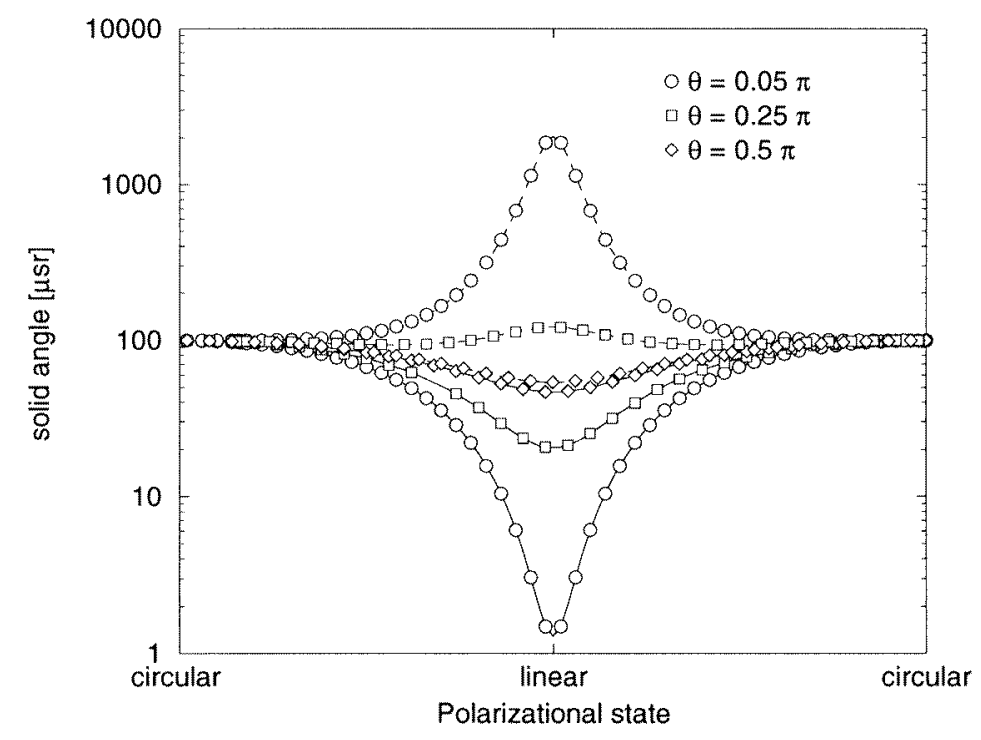

Figure 1. Maximum (broken curves) and minimum (full curves) errors of orientation for various source positions.

At certain times LISA is insensitive to a given linear polarization emitted by sources close to or at the poles. Even for a one-year integration time this leads to a considerable increase in uncertainty. In contrast, such zeros in the sensitivity do not exist for circularly polarized gravitational waves, leading to the observed insensitivity to the source's position.

To obtain a result independent of the binary's orientation, we averaged over every possible orientation. Therefore, figure 2 gives the averaged error of orientation as a function of the declination of the source, $0^{\circ}$ denoting the ecliptic and $\pm 90^{\circ}$ the poles.

The averaged error of orientation increases towards the pole which is clearly an effect of the poor resolution for linearly polarized gravitational waves described above. However, the variations with the azimuth of the source are small compared to the overall variations, giving a distribution close to rotational symmetry.

It should be noted that in contrast with the results obtained by [2] for the angular resolution, the resolution for the orientation of a binary is best for sources located in the ecliptic and decreases towards the poles. 


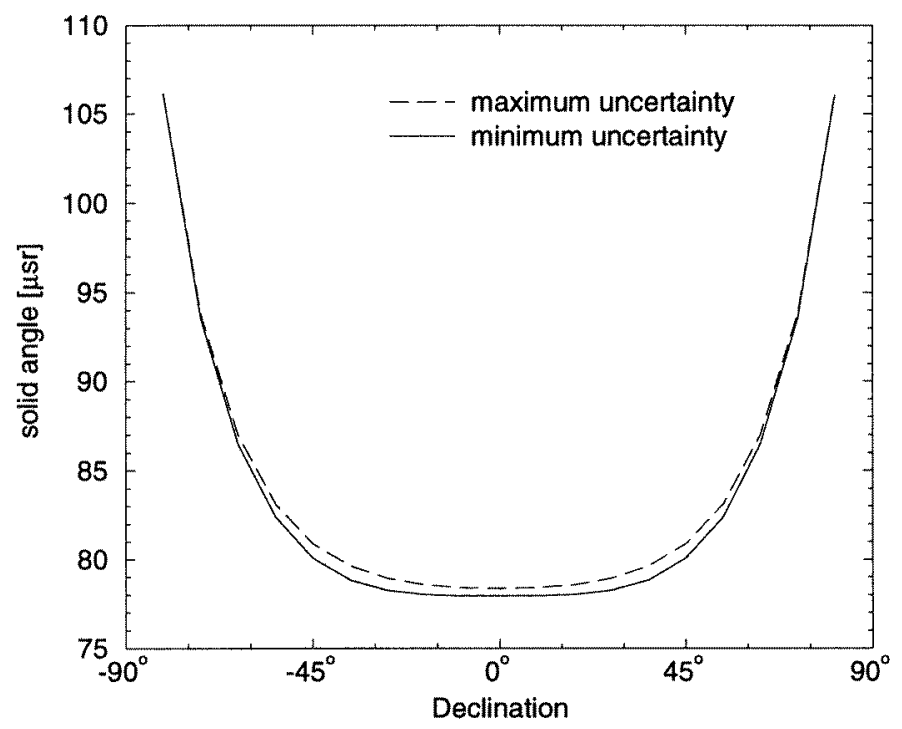

Figure 2. Averaged error of orientation as a function of source declination. Variations with the azimuth are small compared to overall variations.

\section{Conclusion}

Using the method of Gaussian approximation, we obtained $110 \mu \mathrm{sr}$ at a signal-to-noise ratio of 100 as an upper limit for the uncertainty of the orientation of a binary observed by LISA. Although restricted to monochromatic binaries at a frequency of $3 \mathrm{mHz}$ with circular orbits, it is clear that this result will encourage the use of LISA as an astronomers' instrument.

\section{References}

[1] Peterseim M, Jennrich O and Danzmann K 1996 Class. Quantum Grav. 13 A279-84

[2] Peterseim M, Jennrich O, Danzmann K and Schutz B F 1997 Class. Quantum Grav. 14 1507-12

[3] Danzmann K et al 1996 LISA Pre-Phase A Report MPQ-Report 208, Max-Planck Institut für Quantenoptik, Garching

[4] Cutler C and Flanagan E E 1994 Phys. Rev. D 49 2658-97

[5] Gürsel Y and Tinto M 1989 Phys. Rev. D 40 3885-995

[6] Finn L S 1992 Phys. Rev. D 46 5236-49

[7] Thorne K 1989300 Years of Gravitation ed S W Hawking and W Israel (Cambridge: Cambridge University Press) 